\title{
Researching CdZnS/ZnS (GT) Quantum Dots in Cathodoluminescent Mode
}

\author{
Majmaa Huda Khalid Hameed, Sheshin E.P
}

\begin{abstract}
Application field of UV light sources is getting larger at the last decades. Among the most widespread are high and medium pressure vacuum lamps. But there currently is a trend of moving away from using mercury both in household applications and manufacturing. This creates a necessity to conduct research and development for $\mathrm{UV}$ sources made and operating without $\mathrm{Hg}$. Cathodoluminescent $U V$ sources are in this category. One of the possible ways to create a viable $U V$ anode phosphor is using quantum dots with needed spectral characteristics.
\end{abstract}

Keywords: $U V$ light, light sources, quantum dots, cathodoluminescence, field effect emission, CdZnS/ZnS

\section{INTRODUCTION}

A cathodoluminescent emission source is a vacuum lamp, either of diode-like or triode-like design, with an electron gun and an anode screen covered with an anode phosphor. The anode phosphor is selected beforehand to be activated by fast electrons (5-30 keV). Because of the lamp spectrum being dependent only on the type of anode phosphor used, same design could be applied to UV light sources creation, by changing the anode phosphor. The most efficient design could be achieved by using field emission cathodes instead of thermionic ones. In comparison with field effect cathodes, thermionic cathodes usually require additional energy and time to heat up, and have lower working time. A field effect vacuum lamp could, in principle, become a new unique UV source. A theoretical maximum of anode phosphor energy efficiency is 36-40\% [1]. Practical results show a number about $25 \%$ for blue spectrum and even less when considering longer waves. So it seems reasonable to expect efficiency about $30 \%$ for UV spectrum.
Revised Manuscript Received on July 18, 2019.

Majmaa Huda Khalid Hameed, Moscow Institute of Physics and Technology, 141709 Institutsky Lane, Dolgoprudny, Moscow District, Russian Federation

Sheshin E.P, Moscow Institute of Physics and Technology, 141709 Institutsky Lane, Dolgoprudny, Moscow District, Russian Federation.

\section{QUANTUM DOTS BASIC INFORMATION}

One of the possible ways of creating UV phosphors with necessary spectrum characteristics is using quantum dots (QD). The emission spectrum of these semiconductor nanocrystals depends on the nanocrystal size, with the spectrum of an isolated nanocrystal consisting of a single very thin line [2]. It is therefore possible to create an anode phosphor with a single line 1-2 $\mathrm{nm}$ wide. It has been proven experimentally that multi-shell QDs can be used as anode phosphors, showing effective activation from an electron beam. The emission brightness is comparable to classical anode phosphors of the same spectrum [3], with efficiency being $\sim 10-20 \%$ even with quantum yield being far from $100 \%$.

The main requirements for QD source materials are the following:

1. Straight-zone spectrum - allows for efficient luminescence,

2. Small effective mass of charge-carrying particles quantum effects must be exhibited in a range of sizes wide enough (at nanoscale).

3.

Apart from composition and size, the form of QDs effects their properties considerably:

Spherical - Most of the QDs have spherical form. This family of quantum dots nowadays has the broadest range of applications. Spherical QDs are the easiest to produce.

- $\quad$ Ellipsoidal - nanocrystals are oblong at one of their axis.

- Complex geometry nanocrystals. It is possible to synthesize crystals having rather diverse complex geometry variants: cuboids, astroids, branched fractal structures and so on.

For our purposes, the most interesting are QDs capable of photoluminescence: quantum dots, in which photon absorption causes a birth of an electron-hole pair, with its recombination resulting in fluorescence. Such quantum dots usually have a thin and symmetrical fluorescence peak in their spectrum, with the peak position determined by QD size. As of such, depending on size and composition, quantum dots can have fluorescence in UV, visible light, or in IR.

Having the above information in mind, it was deemed appropriate to conduct a comparative analysis of quantum dots with similar structure: $\mathrm{CdZnS} / \mathrm{ZnS}$ and $\mathrm{CdZnS} / \mathrm{ZnS}$ (GT), both emitting at $390 \mathrm{~nm}$, and being used as an anode phosphor. The two specimen types differ by a lifeextending silicon-organic 
modifier being present or absent in the compound [4].

\section{EXPERIMENTAL \& RESULTS}

Specimens of CdZnS/ZnS and CdZnS/ZnS (GT) quantum dots if the form of toluene colloid solution were purchased and applied to an ITO conducting glass substrate via sedimentation method. The specimens were then dried at $80^{\circ} \mathrm{C}$ for 4 hours. A specimen of each type was taken to a REM imaging research in order to control application uniformness (Fig. 1).

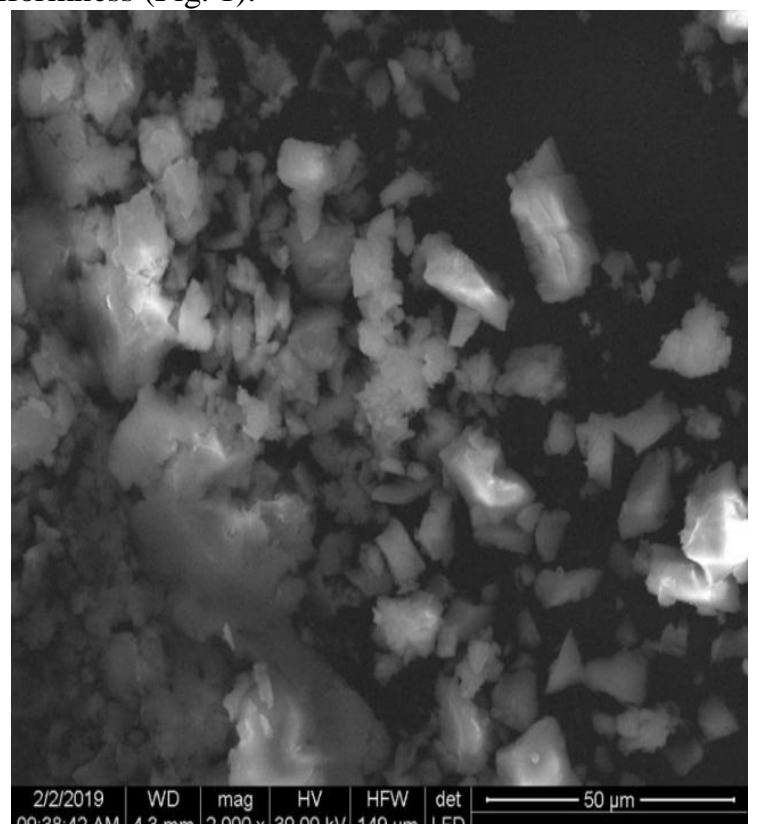

Fig. 1. REM image of a CdZnS/ZnS (GT) specimen.

After that, the specimens were mounted at a vacuum flange (Fig. 2) and were put at the vacuum chamber, where at a residual gas pressure of $\sim 10^{-6}$ Topp the research took place. At anode voltage of $6.7 \mathrm{kV}$ both types of specimens showed stable activation under field effect electron current. Cathode current for each specimen was kept at about $1 \mathrm{uA}$.

The $\mathrm{CdZnS} / \mathrm{ZnS}$ emission was too weak to be reliably picked up by the available spectrometer. the $\mathrm{CdZnS} / \mathrm{ZnS}$ (GT) was too weak for a detailed spectrum research, but was well visible from vacuum chamber walls rereflection. The experiment was being conducted for 15 minutes for $\mathrm{CdZnS/ZnS} \mathrm{(GT),} \mathrm{when} \mathrm{the} \mathrm{observed} \mathrm{rereflected} \mathrm{emission}$ intensity dropped dramatically, and any subsequent attempts to achieve noticeable reactivation did not succeed, despite anode current present. Such a phenomenon also was registered for $\mathrm{CdZnS} / \mathrm{ZnS}$, but the intensity drop (total absence of any visible radiation) happened in less than a minute. The effect was stably observed with all the subsequent experiments. This fact could be formally interpreted as quantum dots degradation under the electron current.

A specimen of the same type undergone an attempt to increase anode current up to $100 \mathrm{uA}$ by raising anode voltage to $15 \mathrm{kV}$. This way, not only electron beam intensity was increased, but electrons energy as well. This, still, resulted in the same low intensity for both specimens with no significant change in radiation patterns, which may indicate insufficient QD concentration or perhaps show that specimens go into saturation at much lower currents than that.

As of this, the QD specimens researched show rather low operation life and low radiation intensity, which allows to conclude that further research may be impractical. Despite that, it is worth noticing that introducing silicon-organic additives leads to an increase in both specimens' life and radiation intensity. A possible explanation is the increase in shell size of quantum dots leads to better durability under electron bombardment.

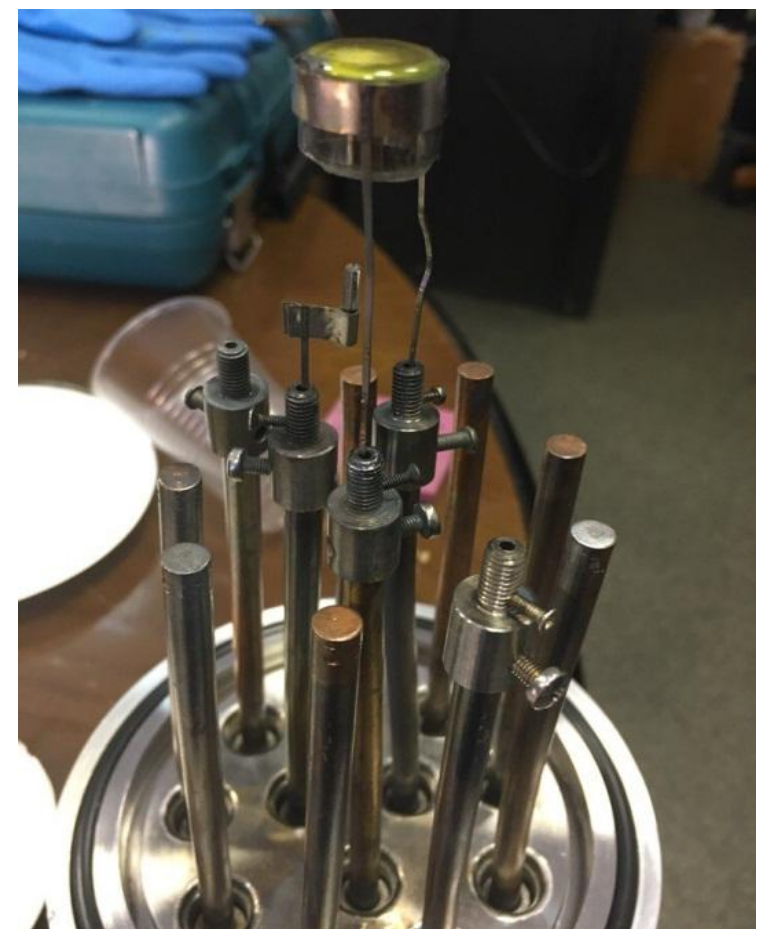

Fig. 2. CdZnS/ZnS (GT) specimen just before mounting the flange to a vacuum chamber

\section{CONCLUSION}

Despite QDs being rather perspective as a type of anode phosphor, these experiments show that operating life of quantum dots may still be insufficient in such an application. Also, according to experimental data, using silicon-organic modifiers can yield an increase in operating life, which may be used in future research and development for more perspective designs. We assume the insufficient operating time to be caused by the number of QD shells (their size) being too small, and the rather low radiation intensity to be connected to low concentration of the dots in raw solution, which led to insufficient amount of QDs in the resulting specimens.

\section{REFERENCE}

1. Levshin V.L. et al. Researching cathodoluminescence of $\mathrm{ZnS}$ and some other phosphors // Works of P.N. Lebedev's Institute of Physics. 1963. \#23. P. 64-135.

2. Klimov V.I., ed. Nanocrystal Quantum Dots (2nd Ed.). // CRC Press, 2010

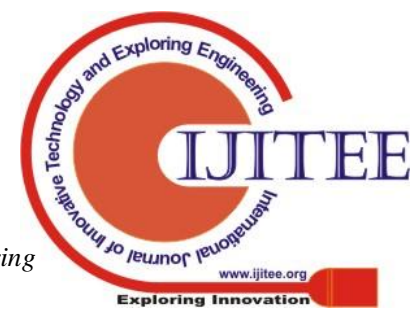


3. Ozol D.I. Preliminary study of cathode ray tube phosphors on the basis of nanocrystal quantum dots // 29th International Vacuum Nanoelectronics Conference (IVNC), 2016, Vancouver, BC, 2016, pp. 1-2.

4. Dezhurov S.V. et al. Synthesizing highly stable colloid quantum dots CDTESE/CDS/CDANS/ZNS, exhibiting fluorescence in 650-750 $\mathrm{nm}$ range // Russian nanotechnology, 2016, \#5-6 pp. 69-74 